\title{
Wind accretion in Cygnus X-1
}

\author{
E. Meyer-Hofmeister ${ }^{1}$, B. F. Liu ${ }^{2,3}$, E. Qiao ${ }^{2,3}$, and R. E. Taam ${ }^{4}$
}

\author{
1 Max-Planck-Institut für Astrophysik, Karl-Schwarzschildstr. 1, 85740 Garching, Germany \\ e-mail: emm@mpa-garching.mpg.de \\ 2 Key Laboratory of Space Astronomy and Technology, National Astronomical Observatories, Chinese Academy of Sciences, \\ Beijing 100012, PR China \\ 3 School of Astronomy and Space Science, University of Chinese Academy of Sciences, 19A Yuquan Road, Beijing 100049, \\ PR China \\ ${ }^{4}$ Center for Interdisciplinary Exploration and Research in Astrophysics (CIERA), Department of Physics and Astronomy, \\ Northwestern University, 2145 Sheridan Road, Evanston, IL 60208, USA
}

Received 23 January 2020 / Accepted 20 March 2020

\begin{abstract}
Context. Cygnus X-1 is a black hole X-ray binary system in which the black hole captures and accretes gas from the strong stellar wind emitted by its supergiant $\mathrm{O} 9.7$ companion star. The irradiation of the supergiant star essentially determines the flow properties of the stellar wind and the X-ray luminosity from the system. The results of three-dimensional hydrodynamical simulations of wind-fed $\mathrm{X}$-ray binary systems reported in recent work reveal that the ionizing feedback of the X-ray irradiation leads to the existence of two stable states with either a soft or a hard spectrum.

Aims. We discuss the observed radiation of Cygnus X-1 in the soft and hard state in the context of mass flow in the corona and disk, as predicted by the recent application of a condensation model.

Methods. The rates of gas condensation from the corona to the disk for Cygnus X-1 are determined, and the spectra of the hard and soft radiation are computed. The theoretical results are compared with the MAXI observations of Cygnus X-1 from 2009 to 2018 . In particular, we evaluate the hardness-intensity diagrams (HIDs) for its ten episodes of soft and hard states which show that Cygnus X-1 is distinct in its spectral changes as compared to those found in the HIDs of low-mass X-ray binaries.

Results. The theoretically derived values of photon counts and hardness are in approximate agreement with the observed data in the HID. However, the scatter in the diagram is not reproduced. Improved agreement could result from variations in the viscosity associated with clumping in the stellar wind and corresponding changes of the magnetic fields in the disk. The observed dipping events in the hard state may also contribute to the scatter and to a harder spectrum than predicted by the model.
\end{abstract}

Key words. X-rays: binaries - binaries: close - stars: individual: Cygnus X-1 - stars: winds, outflows

\section{Introduction}

Cygnus X-1 is one of the most frequently observed high-mass X-ray binaries (HMXB) having been studied with instruments on ground-based and space-based telescopes. The source consists of a compact object, a black hole, and a blue supergiant star, HDE 226868, in close proximity, orbiting about their common center of mass with a period of 5.6 days (for a review of parameters, see Orosz et al. 2011). A fraction of the mass lost from the $\mathrm{O} / \mathrm{B}$ star is likely accreted in the form of a coronal flow possibly forming an inner accretion disk, which results in either a hard or soft X-ray spectrum. The observations of Cygnus X-1 (hereafter Cyg X-1) over several decades record the long-lasting hard and soft spectral states. Based on the extensive observational database, many theoretical investigations were carried out to analyze the distinct features of Cyg X-1. In particular, the spectra in the hard and soft states and the change between spectral states have been observationally studied on the one hand, while the formation and structure of the wind of the O/B star, including the influence of irradiation have been theoretically studied on the other. Furthermore, studies of the similarities and differences in comparison with transient X-ray binaries (e.g., GX 339-4), the short-time and long-time variability, and the presence of radio emission and $\gamma$-ray emission have been undertaken.
The investigation of the structure and properties of stellar winds from massive early-type stars, as in the case of the binary Cyg X-1, was pioneered by the work of Castor et al. (1975). The model describes the mass loss driven by line absorption and scattering of the supergiant radiation field (for a review see Čechura \& Hadrava 2015). Recently, the influence of photoionization caused by the feedback of X-ray radiation from the compact companion, black hole, or neutron star was taken into account and a new radiation hydrodynamic model was developed by Čechura \& Hadrava (2015) and applied to the known HMXBs, especially to Cyg X-1. The three-dimensional numerical simulations provide a framework for the description of the wind flow between the two binary components during the two spectral states for X-ray luminosities of $1.9 \times 10^{37} \mathrm{erg} \mathrm{s}^{-1}$ and $3.3 \times 10^{37} \mathrm{erg} \mathrm{s}^{-1}$ as deduced from observations. As the observations show, these two states are stable and exhibit some scatter in the hardness and count rate. Important for the description is the influence of clumping, as found in recent work by Krtička et al. (2018).

Given the existence of the states characterized by (i) a low luminosity with a hard spectrum and (ii) a high luminosity with a soft spectrum, we examine the nature of the accretion process in the innermost region of the disk surrounding the black hole. Specific attention is focused on the distribution of the mass flow 
in the corona and disk in this region. In a recent study Taam et al. (2018) proposed a new accretion picture for Cyg X-1 where the condensation of gas from the corona onto a small or larger inner disk determines the spectrum in the hard and soft state. It was shown that the spectrum was dependent on the rate of mass captured from the companion star through the corona. In this paper, we aim to clarify whether the spectral changes documented by the long-time observations validate this accretion model.

Here we use the data of Cyg X-1 obtained with the all-sky monitor MAXI on board the Japanese module of the International Space Station (Matsuoka et al. 2009). The observations during the years from 2009 to 2014 have been analyzed in the investigation of the long-term variations in the low/hard and in the high/soft state by Sugimoto et al. (2016). Our investigation is a continuation and extension of their study; we include the data through December 2018 with the goal of focusing on the evolution of the spectral states for comparison with the new accretion model for Cyg X-1.

In Sect. 2 the light curves of Cyg X-1 from the MAXI observations are obtained for various energy bands to illustrate the repetitive phases between the hard and soft spectral states for the years between 2009 and 2018. During this period, the source evolved through ten episodes, each consisting of a hard spectral state followed by a soft state. In Sect. 3 the hardness-intensity diagrams (HIDs) are shown for the episodes characterized by long durations. Since the hot wind from the companion star is an essential feature of the accretion model for Cyg X-1, a short review of the theoretical results for hot star winds, especially of the influence of irradiation on the ionization structure of the winds, is presented in Sect. 4. Of particular importance are the results of the three-dimensional time-dependent radiation hydrodynamic simulation of the stellar wind by Čechura \& Hadrava (2015) and the influence of small-scale density inhomogeneities (clumping) on the strength of the wind. In Sect. 5 we discuss how the states of low and high luminosity (which differ by only a factor of two) and the spectral hard and soft states can be understood within the framework of the model of Taam et al. (2018) for the distribution of mass flows near the black hole. In Sect. 6 the observed dipping events as an external cause for scatter in the hardness intensity diagrams are discussed. Finally, we conclude in Sect. 7.

\section{MAXI observations of Cyg X-1}

Our investigation is based on observations obtained during the years from 2009 to 2018. Following the analysis of Sugimoto et al. (2016), the archival one-day-bin data of Cyg X-1 can be downloaded from the MAXI home page ${ }^{1}$. Very recently, it was brought to our attention that the latest processed data for Cyg X-1, where data affected by the interference of the ISS solar panels are excluded, will be available on the homepage (priv. comm.). With fewer zero or negative flux values in these newly processed data, the light curves are displayed for the four energy bands in Fig. 1. These light curves clearly show the two different spectral states.

To study the HIDs the older data processed with version $5 \mathrm{~L}$ of the data analysis package are used. We note that the photon counts with values near or less than zero are not used in the distribution of points in the HIDs.

In Sugimoto et al. (2016) a hardness, $h$, defined as the ratio of counts in the energy bands $4-10 \mathrm{keV}$ and $2-4 \mathrm{keV}$, and an

\footnotetext{
1 http://maxi .riken.jp/top/index $\cdot$ php?cid=1\&jname= J1958+352
}

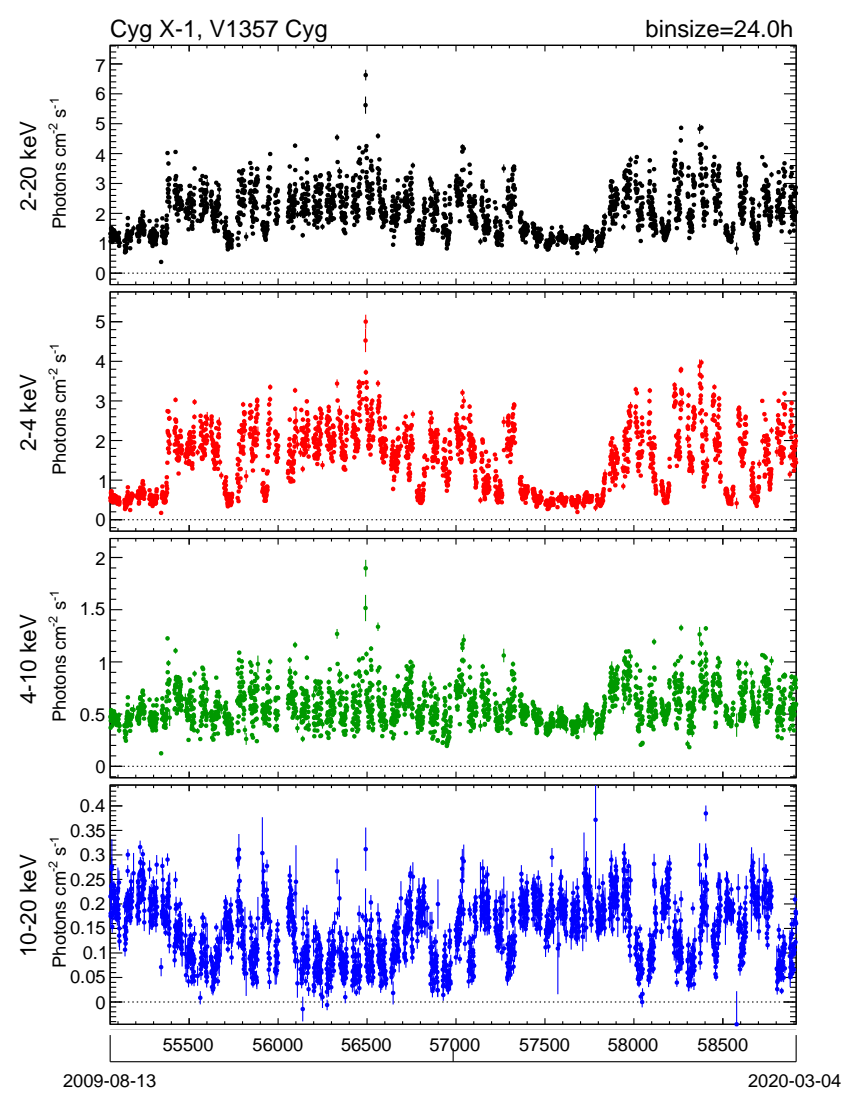

Fig. 1. One-day-bin light curves of Cyg X-1 obtained with MAXI/GSC, processed by the latest pipeline (priv. comm.).

intensity of counts in the energy band $2-10 \mathrm{keV}$ were used. The spectral states were defined as follows: high soft state (HSS) if $h<0.43$ and low hard state (LHS) if $h>0.48$. As documented by Sugimoto et al. (2016) in their Fig. 2 only $1 \%$ of the counts were in the intermediate hardness range between the two states during all the years from 2009 to 2014 . The variability from day to day is remarkable, and its analysis has been the subject of many investigations. It should be noted that data from previous and current all sky monitors on RXTE, Swift-BAT, and FermiGBM have been also used for studies of the spectral states of Cyg X-1 (Grinberg et al. 2013). A long-term monitoring campaign of Cyg X-1 with RXTE from 1999 to 2011 (Grinberg et al. 2014) provided timing data to establish the relation between its timing properties and spectral states.

\section{Hardness-intensity diagrams}

The statistical analysis of the observations by Grinberg et al. (2013) has shown that there is a high probability for Cyg X-1 to remain in a hard or soft state. Theoretically, the results of the time-dependent hydrodynamical simulations of stellar wind-fed $\mathrm{X}$-ray sources reveal that there are two solutions at luminosities corresponding to the hard and soft spectral state (for details, see the following section). This agreement with the observations suggests that the two states are stable. A graphical representation of the data in an HID provides a clear picture of the distribution of observations in these two states.

The HID of all observations of Sugimoto et al. (2016, Fig. 2) reveals points scattering about a hardness value of about 0.26 in the soft state and 0.78 in the hard state. In their Table 2, five episodes of a hard state followed by a soft state are listed. 


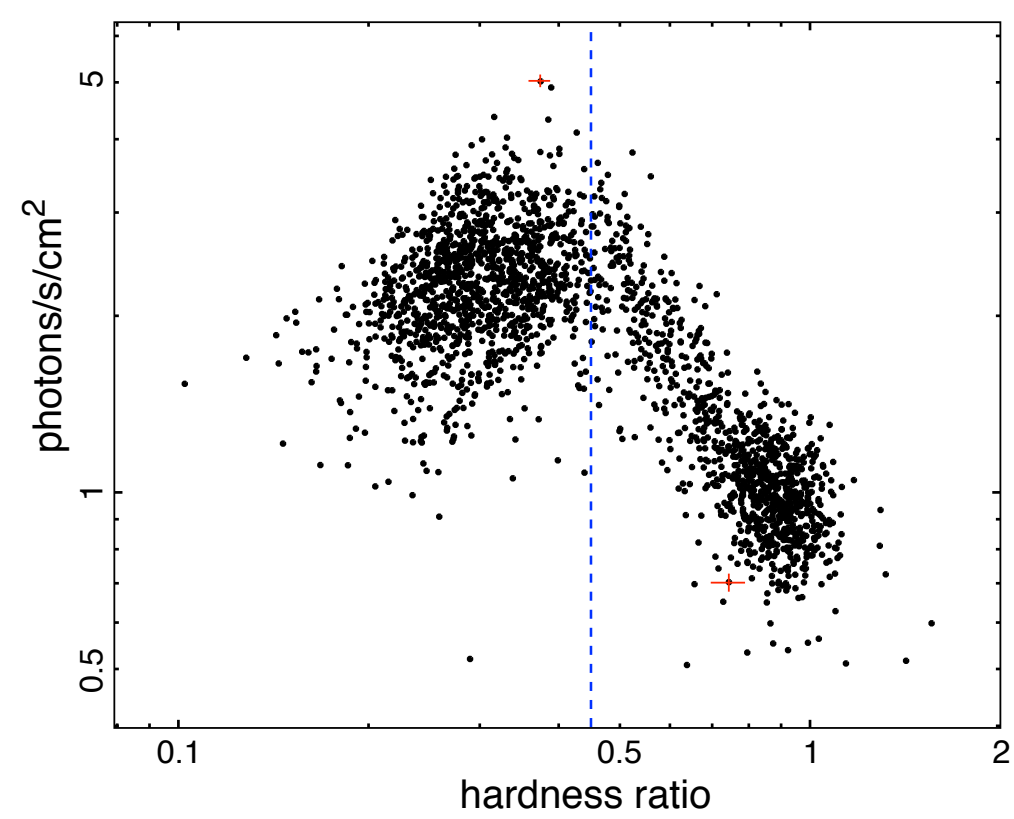

Fig. 2. Hardness-intensity diagram for all MAXI observations during the time period from 2009 to 2018 for Cyg X-1. Photon counts in the energy band $2-10 \mathrm{keV}$ are approximated by counts in the band $2-20 \mathrm{keV}$ minus counts in $10-20 \mathrm{keV}$. The hardness ratio is defined as counts in the energy bands $4-10 \mathrm{keV}$ vs. $2-4 \mathrm{keV}$. The blue vertical line indicates the threshold of $h=0.45$. Two red error bars indicate the errors at higher and lower photon count rates.
In Fig. 2 we show the HID for all observations in the years from 2009 to 2018 . Since the photon counts in the energy band $2-10 \mathrm{keV}$ are not available, we used the counts in the band 2-20 keV minus counts in $10-20 \mathrm{keV}$. We show representative error bars in Fig. 2 taken from Fig. 2 in Sugimoto et al. (2016). The two error bars document the larger errors at points of low count rates and smaller errors at points of higher count rates.

During the long-term MAXI observations in which we use the hardness ratio of counts in the energy bands $4-10 \mathrm{keV}$ and 2-4 keV, ten episodes of a hard spectral state followed by a soft spectral state are found. In Table 1 the duration of the episodes is listed. These data processed using version $5 \mathrm{~L}$ of the data analysis package only led to small changes compared to the processed data used earlier. We take the observations with $h<0.45$ as the soft state and with $h>0.45$ as the hard state, adjusting the definition according to that of Sugimoto et al. (2016; where they excluded the data of extremely rare hardness values in the range from 0.44 to 0.47 as intermediate state). For the long-term observations used here the threshold between hard and soft state is only approximate and is not as clear as in the data used by Sugimoto et al. (2016). This is caused by the data in episode 8 (see Fig. 3).

The episodes last for varying time intervals and the distribution of photon counts and hardness in the HID differ during the individual episodes. The different duration of the hard and soft state displays a random character of the transitions. In Fig. 3 the HIDs for the four episodes with relatively long durations in both spectral states are shown.

The accretion process during the hard spectral state of Cyg X-1 was often compared with that in low-mass X-ray binaries (LMXBs) in the hard state, especially GX 339-4. For comparison, in Fig. 4 we show an HID for the outburst of GX 339-4 in 2010. The data are based on MAXI observations from December 2009 to June 2011 (MAXI homepage, data products version L-6). The hardness is determined using the same energy bands as for Cyg X-1. The HID shows the data, as the source evolves, from the hard state of low and then increasing intensity to the transition to the soft state and the decreasing intensity during the soft state with a return to the hard state. Upon inspection of the full cycle at both high and low luminosities (similar for other outbursts) the difference is clear: in LMXBs the luminosity
Table 1. Spectral states of Cygnus X-1 during the MAXI observations 2009-2018: data and duration of ten episodes of hard state (hardness ratio $h>0.45)$ and soft state $(h<0.45)$.

\begin{tabular}{|c|c|c|c|c|c|c|}
\hline \multirow[t]{2}{*}{ No. } & \multicolumn{2}{|c|}{ Hard state } & \multicolumn{4}{|c|}{ Soft state } \\
\hline & $\begin{array}{l}\text { Start } \\
\text { (MJD) }\end{array}$ & $\begin{array}{l}\text { End } \\
\text { (MJD) }\end{array}$ & $\begin{array}{l}\text { Dur. } \\
\text { (d) }\end{array}$ & $\begin{array}{l}\text { Start } \\
\text { (MJD) }\end{array}$ & $\begin{array}{l}\text { End } \\
\text { (MJD) }\end{array}$ & $\begin{array}{l}\text { Dur. } \\
\text { (d) }\end{array}$ \\
\hline 1 & 55057 & 55378 & 321 & 55379 & 55673 & 294 \\
\hline 2 & 55680 & 55789 & 109 & 55790 & 55886 & 96 \\
\hline 3 & 55911 & 55941 & 30 & 55942 & 56039 & 97 \\
\hline 4 & 56040 & 56077 & 37 & 56078 & 56732 & 654 \\
\hline 5 & 56733 & 56746 & 13 & 56747 & 56757 & 10 \\
\hline 6 & 56780 & 56839 & 59 & 56854 & 57104 & 250 \\
\hline 7 & 57105 & 57268 & 163 & 57269 & 57331 & 62 \\
\hline 8 & 57358 & 57968 & 610 & 57969 & 58111 & 142 \\
\hline 9 & 58112 & 58201 & 89 & 58205 & 58387 & 182 \\
\hline 10 & 58388 & 58411 & 23 & 58441 & 58480 & 39 \\
\hline
\end{tabular}

increase and decrease is much more than a factor of ten (outbursts last months) and quiescence intervals last several years. In Cyg X-1 the variation in luminosity is about a factor of two and only a moderate soft state is reached. A similar distinction was found by Belloni et al. (2010, Fig. 8) for RXTE/PCA observations of Cyg X-1 from 1996 to 2005 in comparison with observations of the 2002-2003 and 2004-2005 outbursts of GX 339-4 (where the hardness is defined as the ratio of counts in the energy bands $6.3-10.5 \mathrm{keV}$ and $3.8-6.3 \mathrm{keV}$ according to RXTE/PCA). Another striking difference between the HIDs of LMXBs and Cyg X-1 is the appearance of a hysteresis during the luminosity decrease which is present only for LMXBs.

The different spectral features as shown in the HID support the view that the accretion process in Cyg X-1 differs from that in LMXBs. This has been studied by Taam et al. (2018), where the gas is supplied via a stellar wind, in contrast to Roche lobe overflow (RLOF), which plays a crucial role in the formation of a different accretion configuration. The enhanced gas supply via a stellar wind leads to a perpetual existence of an inner disk and 

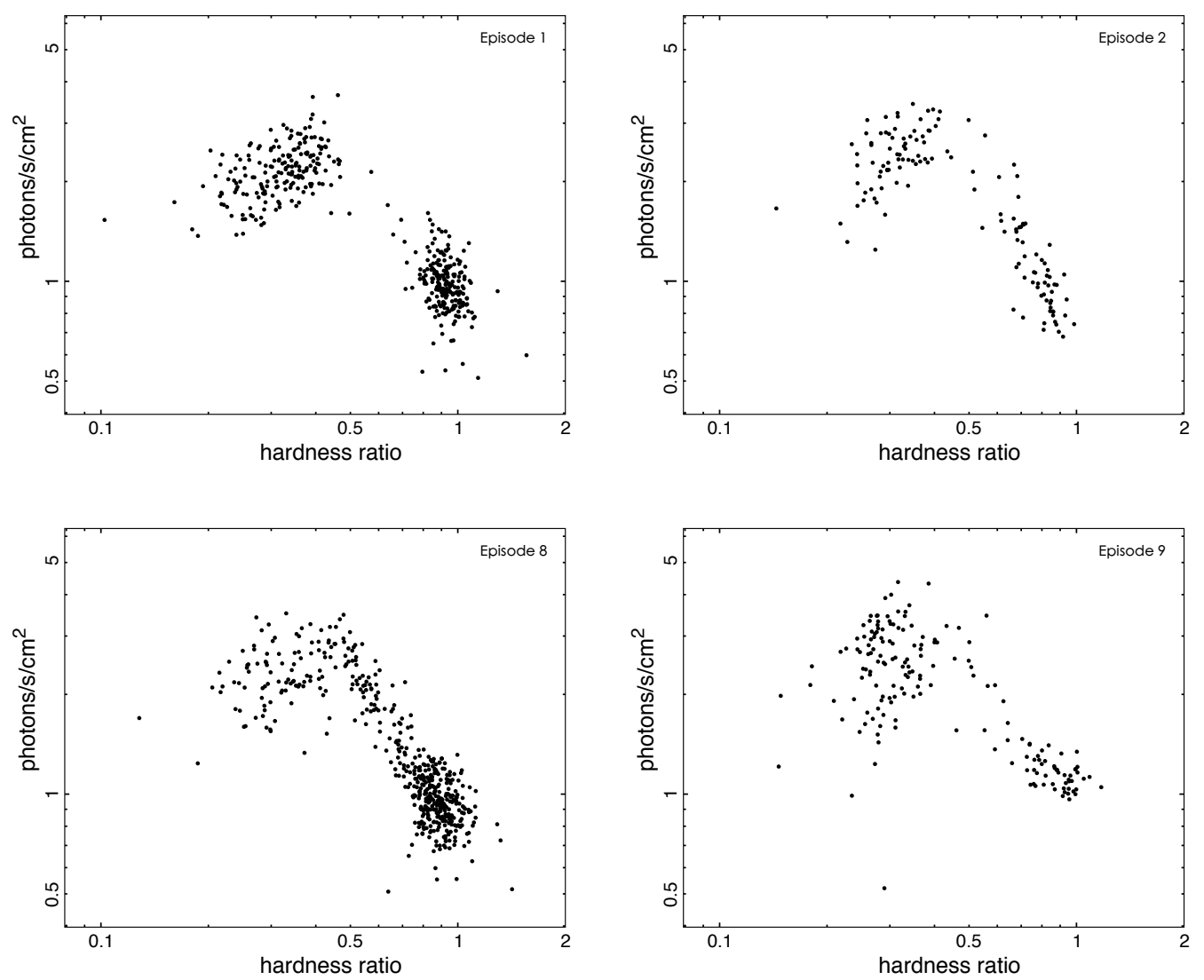

Fig. 3. Hardness-intensity diagrams for episodes 1, 2, 8, and 9 for the hard and soft states; see the time intervals listed in Table 1. Hardness is defined as in Fig. 2.

perpetual soft irradiation in contrast to the situation during the rise to an outburst in LMXBs where an ADAF fills the region near the black hole and hard irradiation leads to the hysteresis (Meyer-Hofmeister et al. 2005).

\section{Properties of the stellar wind from massive early-type stars}

\subsection{Influence of $X$-ray irradiation on the wind in Cyg X-1}

An understanding of the soft and hard X-ray radiation emitted by Cyg X-1 as a result of the accretion of hot gas from the companion star requires knowledge of the properties of the stellar wind from massive early-type stars. Models for the description of such winds were developed in the seminal work of Castor et al. (1975, hereafter CAK), followed by elaborations of the theory in several subsequent investigations (for a review, see Čechura \& Hadrava 2015). The mass loss from the early-type donor star occurs in form of a radiatively driven stellar wind modulated by the tidal forces of the compact companion (Hadrava et al. 2013). A code for the three-dimensional time-dependent radiation hydrodynamical simulation was further developed (Čechura \& Hadrava 2015), including the effect of the X-rays emitted from the innermost region near the black hole. It was shown that the feedback effect of the ionizing radiation severely limits the efficiency of the CAK line-driven mechanism and decreases the radiative drag on the wind. As a result, this inhibition slows down the wind causing a broadening of the bow shock that forms in front of the accretion disk surrounding the compact object, thereby increasing the amount of material captured in the accretion disk.

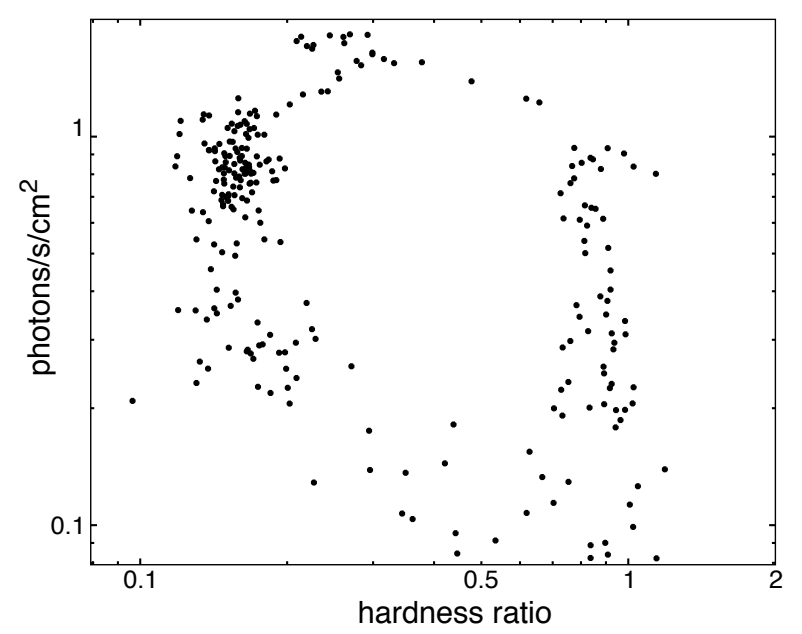

Fig. 4. Hardness-intensity diagram for MAXI observations for GX 339-4 during the outburst in 2010 (observations from December 25, 2009 to June 19, 2011. Photon counts in the energy band 2-10 keV; hardness: counts in the energy bands $4-10 \mathrm{keV}$ vs. $2-4 \mathrm{keV}$.

Hence, the irradiation effect in Cyg X-1 leads to very different wind launching on the two hemispheres of the donor star. Specifically, the outflow from the hemisphere facing the X-ray source is noticeably slower than from the opposite hemisphere lying in the X-ray shadow where the wind can be accelerated without inhibition. This indicates that the X-ray irradiation leads to added accretion, and consequently to an increase in irradiation. However, there must be an upper limit to the irradiation 
since the radiative driving in the irradiated hemisphere is inhibited to a greater degree as the gas approaches a fully ionized state.

\subsection{Two stable states}

In general the possible rates of wind accretion may lie in a wide range; however, for a stable steady state the irradiation luminosity and accretion luminosity must be consistent (for a discussion of this point, see Krtička et al. 2018, Fig. 10). As shown in the results of the numerical simulations by Čechura \& Hadrava (2015) two solutions for the mass flow in Cyg X-1 are found when feedback via irradiation is included. Specifically, solutions are found at a luminosity value of $3.3 \times 10^{37} \mathrm{erg} \mathrm{s}^{-1}$ and a lower value of $1.9 \times 10^{37} \mathrm{erg} \mathrm{s}^{-1}$. It is noteworthy that the observed $\mathrm{X}$-ray luminosities in the low and high state of Cyg X-1 resemble these two values. Further support for these results is provided by comparison of the synthetic Doppler tomograms of theoretically predicted emission with those derived from spectra of Cyg X-1 (Čechura et al. 2015).

The two solutions have quite a different wind flow pattern. In particular, the high-density flow in the vicinity of the $L_{1}$ point is different in the two solutions, which results in a different mass flow rate in the innermost corona, This is of importance for the accretion picture (Taam et al. 2018), as is discussed later.

\subsection{Clumping and variability}

The influence of the X-rays on the wind ionization structure, addressed already in work by Pauldrach (1987), was later reconsidered in more detailed prescriptions for the X-ray radiation feedback (for a review, see Krtička \& Kubát 2016). Specifically, small-scale wind inhomogeneities (Krtička et al. 2018) were taken into account as a new effect. Such clumping in the radiation-driven winds of hot massive stars is understood to arise as a consequence of the line-deshadowing instability (for a review, see Puls et al. 2008; for recent results, see Sundqvist et al. 2018). The clumping weakens the effect of the $\mathrm{X}$-ray irradiation because it favors recombination. By adopting the parameters of a binary, such a wind model can be used to determine the highest possible X-ray luminosity (Krtička et al. 2015) for wind-fed sources. For 21 sources the theoretical results were compared with the observed luminosities. The best match with the observed properties of HMXBs was found for models with radially variable clumping, as suggested by Najarro et al. (2009).

As noted by several authors (Ducci et al. 2009; Fürst et al. 2010; Oskinova et al. 2012), clumping, and therefore fluctuations in the mass accretion rate, was considered a possible cause of the observed X-ray variability (Sugimoto et al. 2016). Since the effect of clumping is smaller during phases of lower irradiation (Krtička et al. 2018) a lower degree of variability in the luminosity from day to day is expected. This can be seen in the light curves (Fig. 1) and is most conspicuous during the longlasting low state from December 2015 to August 2017.

Based on the results of the studies incorporating the ionizing feedback regulated accretion for Cyg X-1 the following picture emerges:

- Two stable states exist at two distinct luminosity levels corresponding to a high luminosity for the soft spectral state and a low luminosity for the hard spectral state. X-ray irradiation is essential in establishing these levels (Krtička et al. 2015). The distribution of points in HIDs, especially of episode 1, suggests that rare large changes in irradiation can initiate a spectral state transition.

- Clumping weakens the effect of irradiation, which may affect the magnetic field and the viscosity, and produces the X-ray luminosity variability on flow timescales of hours to days.

- The strength of clumping is a property of the wind from the $\mathrm{O} / \mathrm{B}$ star and produces a limited range in the luminosity variation.

\section{Spectral properties of the two stable states originating from condensation?}

\subsection{Condensation in Cyg X-1}

The X-ray radiation from the innermost region of the disk, either hard or soft, is determined by the distribution of the mass flow via the corona and the disk, the latter of which is caused by condensation of gas from the corona. A hard spectrum appears if the main accretion flow occurs in the corona as an advectiondominated accretion flow (Narayan \& Yi 1995), and a soft spectrum appears if the main accretion flow is in the disk. The model for the spectral states and the transitions in Cyg X-1 by Taam et al. (2018) describes the two states in terms of the degree to which condensation takes place, as determined by the strength of the wind.

The wind outflow from the companion star as described by the radiation hydrodynamic simulations by Čechura \& Hadrava (2015) gives an explanation for the existence of the two states with differing amounts of mass flow resulting from the radiatively driven wind either originating from the entire surface or only from the unirradiated backside of the O/B star. In this description, the luminosities differ by about a factor of two. In these two states not only is the amount of accretion different, but the structure of the wind flow is also different.

Gas condensation from the corona to the disk is caused by heating of the corona by the viscous dissipation releasing gravitational energy of the accreted gas, and cooling by vertical conduction and inverse Compton scattering of soft photons emitted by the underlying disk (Liu et al. 2015; Qiao \& Liu 2017). This condensation process was already discussed in several investigations for the hard state of LMXBs, where it can lead to the formation of a weak inner disk fed by the condensation of hot gas (Liu et al. 2007, 2011; Meyer et al. 2007; Taam et al. 2008; Qiao \& Liu 2012).

According to the model developed by Taam et al. (2018) the situation in Cyg X-1 differs from that in LMXBs. With matter supplied via RLOF in LMXBs, the outer truncated disk shifts inward to the innermost stable circular orbit when the supply rate increases, leading to a transition from the hard to the soft state. In contrast, the increasing mass supply from a hot wind (only possible with wind accretion such as in Cyg X-1) results in more condensation and a stronger inner disk growing outward, which also leads to a transition to the soft state. The results from the hydrodynamic simulations of Čechura \& Hadrava (2015) reveal the increased high-density flow in the soft spectral state.

\subsection{Results of computations}

The condensation rates are calculated according to the formulae given in the investigation by Liu et al. (2011) and Qiao \& Liu (2012), including the irradiation, which is important for Cyg X1. The results for condensation yield the mass flow rates in the corona and disk in the innermost region. For these mass flow rates spectra were calculated as described in Qiao \& Liu (2017). 


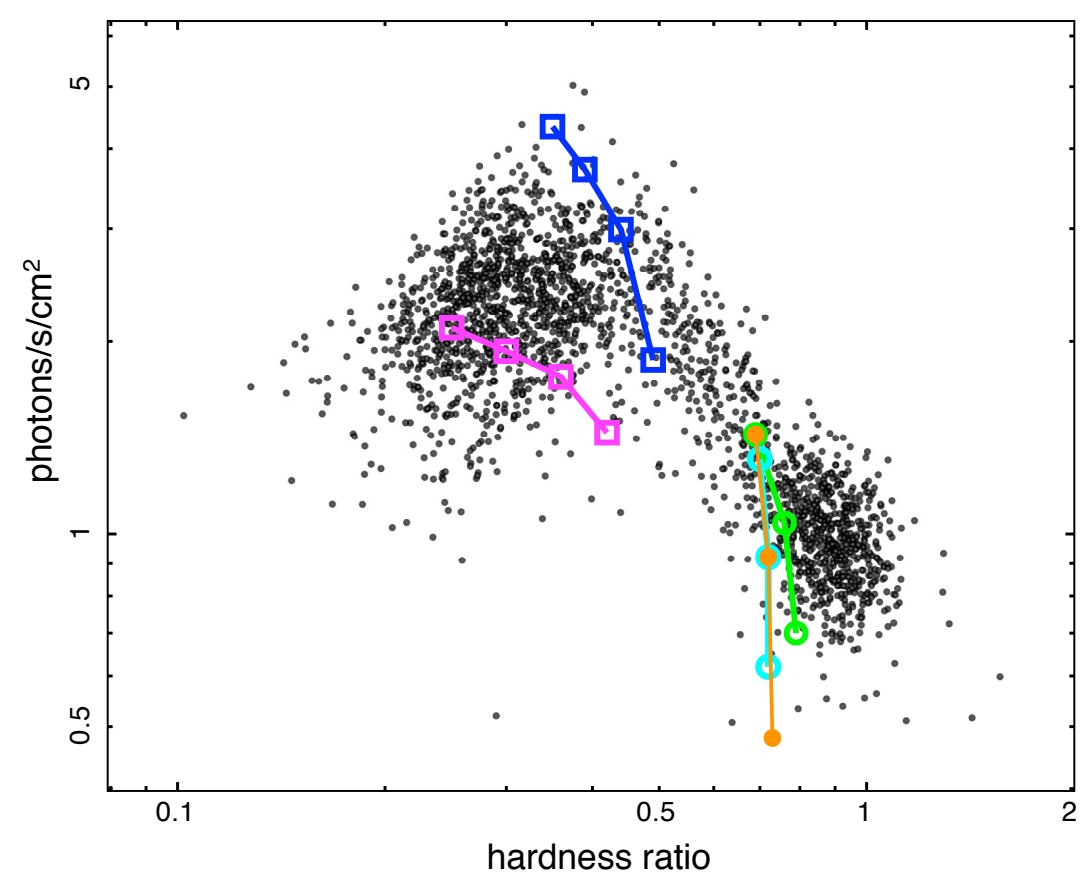

Fig. 5. Comparison of computational results with MAXI observations in Fig. 2. The hardness and count rate are computed from spectra emitted from the disk and corona, as determined by the condensation model. Theoretical results for the soft state are shown for mass supply rates $\dot{M} / \dot{M}_{\mathrm{Edd}}=0.02,0.03,0.04,0.05$ (count rates increase), viscosity $\alpha=0.15$ (purple dots) and $\alpha=0.2$ (blue dots), and albedo fixed at 0.15 . Theoretical results for the hard state are shown for different mass accretion rates $\left(\dot{M} / \dot{M}_{\text {Edd }}=0.03,0.035,0.04\right)$, fixed $\alpha=0.5$, and albedo $=0.5$ (orange dots); for different viscosity $(\alpha=0.45,0.5,0.55)$, a fixed accretion rate of 0.035 , and albedo $=0.5$ (cyan dots); for different albedo $(a=$ $0.5,0.7,0.9)$, fixed viscosity $\alpha=0.5$, and an accretion rate of 0.04 (green dots).
With the spectral energy distribution from the corona and disk the hardness can be determined.

Our primary aim is modeling the radiation in the soft state because the formation of the inner disk due to the mass supply from the hot corona flow is the new explanation suggested by Taam et al. (2018). We computed the condensation of gas in Cyg X-1 for a black hole mass of $15 M_{\odot}$. For our comparison we adopt a series of values for the mass supply rates ranging from 0.02 to $0.05 \dot{M} / \dot{M}_{\text {Edd }}$ (Eddington rate $\dot{M}_{\text {Edd }}=1.4 \times$ $\left.10^{18} M / M_{\odot} \mathrm{g} \mathrm{s}^{-1}\right)$ and values for the viscosity parameter $\alpha=0.15$ and 0.2 and a fixed albedo of 0.15 for the soft state.

The relation between the count rate and hardness is shown in Fig. 5. Typical spectra in the soft state are illustrated in Fig. 6. It can be seen from the spectra that an increase in the mass supply rate leads to a softening of the spectrum and to an increase in the count rate in the $2-10 \mathrm{keV}$ energy band. However, a further increase in the mass supply rate results in a decrease in the count rate with a continuous softening of the spectrum as a consequence of strong condensation at a high accretion rate. This effect is not shown in the figures since Cyg X-1 does not usually reach such a high bolometric luminosity, but it could cause the scattering in the HID at very low hardness and low count rates. The two curves with slightly different viscosity values, $\alpha=0.15,0.2$, indicate a sensitive dependence on the viscosity. Therefore, the variation in the HID can be caused by a change in the mass supply rate accompanied by a change in the viscosity in the accretion flow.

For the hard state, the variation in the count rate and hardness with mass supply rate is studied. In addition, the effects of the viscosity parameter and albedo are also explored. Based on the luminosity in the hard state of Cyg X-1, values for the mass supply rate $\left(\dot{M} / \dot{M}_{\text {Edd }}=0.03,0.035,0.04\right)$ were chosen with a fixed viscosity, $\alpha=0.5$, and albedo, $a=0.5$, which are the same as assumed in the investigation by Taam et al. (2018). As shown in Fig. 5, the count rate decreases steeply with decreasing gas supply rate, while the hardness does not change appreciably. The steep decrease in the photon count rate in the $2-10 \mathrm{keV}$ energy band is caused by the sudden decrease in soft photons from a very weak disk, which largely reduces the effect

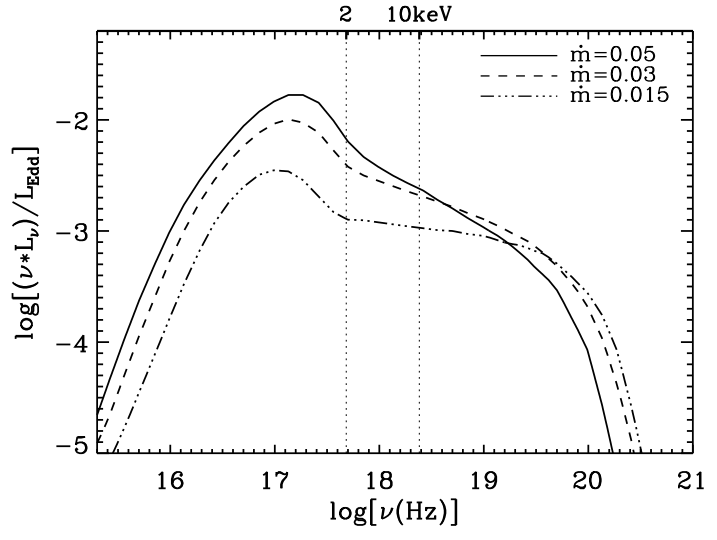

Fig. 6. Spectra for Cyg X-1 in the soft state; condensation determined for a mass supply of $0.015,0.03,0.05 \dot{M}_{\text {Edd }}, \alpha=0.2$, albedo $=0.15$.

of Compton scattering. The weak disk contributes little radiation at the energy band of $2-4 \mathrm{keV}$, leading to less variation in the hardness with accretion rate than that in soft state. These properties in the hard state can be seen from the spectra in Fig. 7. To check the effect of variations in the viscosity and albedo, we first fixed the accretion rate at 0.035 and the albedo at 0.5 , but varied the viscosity parameter slightly, $\alpha=0.45,0.5,0.55$. The results are similar to that obtained by varying the accretion rates. We also calculated the spectrum with $\alpha=0.5$ and $\dot{M} / \dot{M}_{\text {Edd }}=0.04$ fixed while increasing the albedo, $a=0.5,0.7,0.9$, which yields slightly different results. The count rates increase with increasing mass supply, decreasing viscosity, and decreasing albedo, while the hardness only varies slightly from 0.7 .

Upon comparison of the results of the computations with the observations for the years 2009-2018 in Fig. 5, we find that the condensation process can yield photon count rates and hardness in the soft state as observed for Cyg X-1 for the assumed mass supply rates, provided that the viscosity parameter is in the range of $0.1-0.2$. On the other hand, a higher viscosity ( $\alpha \gtrsim 0.5)$ and albedo $(a \gtrsim 0.5)$ might be indicated in the hard state in addition to the contribution from possible obscuration effects (see below). 


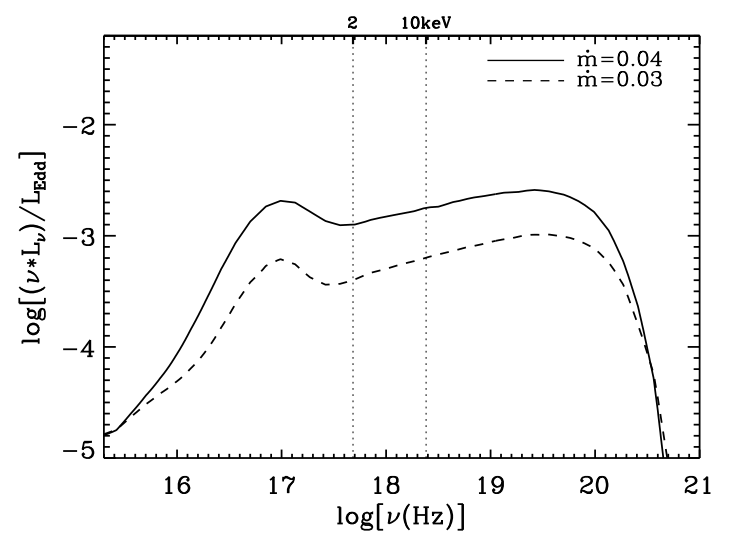

Fig. 7. Spectra for Cyg X-1 in the hard state; condensation determined for a mass supply of $0.03,0.04 \dot{M}_{\mathrm{Edd}}, \alpha=0.5$, albedo $=0.5$.

We note that the observations show a significant scatter in the data. The spread in the distribution of intensity-hardness values may be caused by the mass supply variations due to the expected clumping in the coronal mass flow; we note that the errors in the data are much smaller than this spread. This has been discussed by Krtička et al. (2018), who presume it to be the dominant cause of the variability. However, our results reveal that the condensation process strongly depends on the viscosity leading to larger variations in luminosity and hardness for already small viscosity changes in comparison to that for mass supply variations. It is conceivable that the clumpiness might affect the magnetic field structure in the disk and hence the turbulent viscosity driven by the action of the magneto-rotational instability.

While the theoretical values of the intensity and hardness for the soft state span a range of the observational data the radiation is not as hard as observed in the hard state. A possible explanation could be due to the different wind flow patterns in the soft and hard state found in the hydrodynamical computations. In particular, the high-density flow in the proximity of the $L_{1}$ point could lead to permanent absorption of the radiation from the weak inner disk, so that the observed radiation would appear harder than its intrinsic spectrum. The high-density flow in the proximity of the $L_{1}$ point in the hard state leads to so-called dipping events. However modulations of the column density are found in non-dip spectra as well (Miškovičová et al. 2016). We turn to the dipping events observed in the hard state in the next section.

\section{Dipping events in the hard state}

The scatter in the HID can arise from the dipping events associated with the strong orbital modulation of the column density $N_{\mathrm{H}}$. This modulation results in variable absorption of the $\mathrm{X}$-rays in the stellar wind as was already found in very early work by Li \& Clark (1974) and which has been subsequently studied intensively. Very recently Hirsch et al. (2019) presented an analysis of Chandra High Energy Transmission Gratings observations of Cyg X-1 which point to the picture of a complex wind structure with temperature and density inhomogeneities. As the clumps pass along the line of sight, absorption dips appear in the light curve. During the canonical hard state strong orbital modulation of $N_{\mathrm{H}}$ is observed, but only very little modulation is seen during the thermally dominated X-ray soft state.

It is significant that the apparent difference in the degree to which clumps absorb X-ray radiation in the hard and soft state agrees well with the picture of the wind flow structure resulting from the radiation hydrodynamic simulations of Čechura \& Hadrava (2015, Fig. 9). Specifically, a higher density stream near the black hole is found in the hard state.

We note that the new analysis of Hirsch et al. (2019) was taken during the low hard state. For three sections of the orbital phase with the deepest dipping events, the observed light curve (energy band $0.5-10 \mathrm{keV}$ ) and measured counts in the 0.5$1.5 \mathrm{keV}$ and 3-10 keV energy bands are given with the hardness defined as the ratio of counts in the $0.5-1.5 \mathrm{keV}$ band to the $3-10 \mathrm{keV}$ energy band. During one orbital period the duration of dipping lasts for about one day (first and second dipping) and 1/4 day (third dipping). The daily observations affected by dipping depend on the particular orbital phases observed. If we adopt the results from the Chandra observations, the maximum reduction in the counts could be about $40 \%$ and the hardness could be increased by a factor of two (a rough estimate because of the difference in the definition of the hardness). The possible effect of dipping on the observed photon counts indicates that some of the data points in an HID are shifted to lower intensity and higher hardness. This would affect the degree to which the results from the condensation model compare favorably with the observations for Cyg X-1.

\section{Conclusions}

Cyg X-1 is a particularly conspicuous high-mass X-ray binary with the transfer of matter from its blue supergiant companion to the black hole since the mass transfer rate falls in a range allowing the source to be in two states, with either mainly soft or hard $\mathrm{X}$-ray radiation. The time dependent hydrodynamic simulations of the stellar wind by Čechura \& Hadrava (2015) have shown that X-ray irradiation of the early-type O/B companion leads to a difference in the stellar wind on its two hemispheres. During the hard spectral state of Cyg X-1 the stellar wind is nearly symmetric, whereas during the soft state it is very asymmetric with the matter captured by the black hole mainly launched from the hemisphere lying in the X-ray shadow.

Since the spectrum of the source is determined by the radiation emitted from the innermost region near the black hole, knowledge of the distribution of matter within the corona and the disk is necessary. In this work, the mass flow in the central region depends on the interaction of the cool disk and the hot coronal gas, essentially determined by the condensation process of matter from the corona to the disk. It has already been shown that this hot gas condensation can lead to the formation of a weak inner disk in LMXBs in the hard state.

The condensation process has been applied to the formation of an inner disk in Cyg X-1 during the soft state (Taam et al. 2018), for as strong a mass supply as possible only in wind-fed sources. We compare the results from the condensation model with observations using MAXI data for the years 2009-2019. For this comparison we take the mass flow rates adjusted by condensation; determine the spectra of the radiation, theoretical values of photon count rates, and hardness; and make a comparison with the corresponding values from the observations presented in an HID.

As shown in Fig. 5 the data for Cyg X-1 form two clusters in the HID, one representing the hard state, the other representing a relatively soft state (hardness of photon counts in the energy bands $4-10 \mathrm{keV}$ and $2-4 \mathrm{keV}$ ). This is different from HIDs of LMXBs where the evolution during a single outburst forms a "q-type" loop where the luminosity increases by a factor of more than ten and a hysteresis appears during the return of the source to the low state. 
Upon comparison of theory with bservations, we find agreement with the observations for a mass supply rate of $0.02-0.03$ $\dot{M}_{\text {Edd }}$ and viscosity values of $0.15-0.20$ for the soft state. This confirms that the inner disk can originate from condensation of matter from the hot coronal flow (Taam et al. 2018). The scatter in the HID is not reproduced by the computations, but could be caused by the clumpiness of the mass flow, which can affect the magnetic field structure in the disk and the turbulent viscosity due to the magnetorotational instability.

For the hard state the condensation process leads to spectra that are not as hard as the observed data. The difference may be attributed to the fact that the observations might be affected by absorption in the wind. That is, dips appear in the light curve when clumps in the wind pass along the line of sight. In this case, the observed radiation would appear at higher hardness in the HID for some orbital phases. Such spectral changes during dipping were analyzed by Hirsch et al. (2019) using recent Chandra High Energy Transmission Gratings observations. It should be noted that modulations of the column density in spectra during non-dip events were also found (Miškovičová et al. 2016). It would be highly desirable to carry out studies to examine the possible relationship between the observed high hardness and the effect of obscuration of the inner disk by the wind flow in the hard state.

Acknowledgements. This research has made use of the MAXI data provided by RIKEN, JAXA and the MAXI team. In particular we thank Dr. Mutsumi Sugizaki for providing new data. Financial support for this work is provided by the National Program on Key Research and Development Project (Grant No. 2016YFA0400804), the gravitational wave pilot B (Grant No. XDB23040100), and the National Natural Science Foundation of China (Grant No. 11673026,11773037). We thank Friedrich Meyer and Weimin Yuan for discussions.

\section{References}

Belloni, T. M. 2010, in Lecture Notes in Physics, ed. T. Belloni (Berlin: Springer Verlag), 53, 794

Castor, J. I., Abbott, D. C., \& Klein, R. I. 1975, ApJ, 195, 157

Ducci, L., Sidoli, L., Mereghetti, S., Paizis, A., \& Romano, P. 2009, MNRAS, 398, 2152

Fürst, F., Kreykenbohm, I., Pottschmidt, K., et al. 2010, A\&A, 519, A37

Grinberg, V., Hell, N., Pottschmidt, K., et al. 2013, A\&A, 554, A88

Grinberg, V., Pottschmidt, K., Böck, M., et al. 2014, A\&A, 565, A1

Hadrava, P., \& Čechura, J. 2013 in Feeding Compact Objects: Accretion on All Scales, eds. C. M. Zhang, T. Belloni, M. Méndez, \& S. N. Zhang, IAU Symp., 290, 219

Hirsch, M., Hell, N., Grinberg, V., et al. 2019, A\&A, 626, A64

Krtička, J., \& Kubát, J. 2016, Adv. Space Res., 58, 710

Krtička, J., Kubát, J., \& Krtičková, I. 2015, A\&A, 579, A111

Krtička, J., Kubát, J., \& Krtičková, I. 2018, A\&A, 620, A150

Li, F., \& Clark, G. 1974, ApJ, 191, L27

Liu, B. F., Done, C., \& Taam, R. E. 2011, ApJ, 726, 10

Liu, B. F., Taam, R. E., Meyer-Hofmeister, E., \& Meyer, F. 2007, ApJ, 671, 695

Liu, B. F., Taam, R. E., Qiao, E., \& Yuan, W. 2015, ApJ, 806, 223

Matsuoka, M., Kawasaki, K., Ueno, S., et al. 2009, PASJ, 61, 999

Meyer-Hofmeister, E., Liu, B. F., \& Meyer, F. 2005, A\&A, 432, 181

Meyer, F., Liu, B. F., \& Meyer-Hofmeister, E. 2007, A\&A, 463, 1

Miškovičová, I., Hell, N., Hanke, M., et al. 2016, A\&A, 590, A114

Najarro, F., Figer, D. F., Hillier, D. J., Geballe, T. R., \& Kudritzki, R. P. 2009, ApJ, 691, 1816

Narayan, R., \& Yi, I. 1995, ApJ, 452, 710

Orosz, J. A., McClintock, J. E., Aufdenberg, J. P., et al. 2011, ApJ, 742, 84

Oskinova, L. M., Feldmeier, A., \& Kretschmar, P. 2012, MNRAS, 421, 2820 Pauldrach, A. 1987, A\&A, 183, 295

Puls, J., Vink, J. S., \& Najarro, F. 2008, A\&ARv, 16, 209

Qiao, E., \& Liu, B. F. 2012, ApJ, 744, 145

Qiao, E., \& Liu, B. F. 2017, MNRAS, 467, 898

Sugimoto, J., Mihara, T., Kitamoto, S., et al. 2016, PASJ, 68, S17

Sundqvist, J. O., Owocki, S. P., \& Puls, J. 2018, A\&A, 611, A17

Taam, R. E., Liu, B. F., Meyer, F., \& Meyer-Hofmeister, E. 2008, ApJ, 688, 527

Taam, R. E., Qiao, E., Liu, B. F., \& Meyer-Hofmeister, E. 2018, ApJ, 860, 166

Čechura, J., \& Hadrava, P. 2015, A\&A, 575, A5

Čechura, J., Vrtilek, S. D., \& Hadrava, P. 2015, MNRAS, 450, 2410 